Materials and Methods We performed a prospective study of a SLE pregnant cohort. All patients were evaluated and followed with the same clinical protocol in a multidisciplinary care unit (internal medicine, gynecology, hematology, nephrology) from January 2008 to December 2018. Clinical and laboratory data were collected in a preformed clinical record registry.

Results Seventy pregnancy (67-women) with a medium age of $26(16-41)$ years old were included. Before pregnancy SLE involvement was: cutaneous (75\%), joint (65\%), hematological (41\%) and renal (40\%) (Type -WHO- I:2/II:9/III:3/ IV:5/V:9). Eleven patients had carried out a preconception counselling. Median time between SLE onset and pregnancy was $6(1-25)$ years. At the beginning of the follow-up, disease was active in $15,7 \%$ and status of antibodies was: persistently positive antiphospholipid: $32.8 \%$, anti-SSA/Ro: 24\%, anti-SSB/La: 10\%. Mycophenolate, enalapril and warfarin treatment was suspended at the first visit. During pregnancy treatment included: corticosteroids (63\%), azathioprine (43\%), hydroxychloroquine (97\%), low-molecularweight-heparin (48\%) and low-dose aspirin (74\%). Thirtytwo patients had SLE-flare and 19 (27\%) preeclampsia. Preterm delivery (PD) was $42.9 \%$. SLE-flare during pregnancy was associated with $\mathrm{PD} \quad(\mathrm{p}=0.00)$ and preeclampsia $(p=0.04)$. Lupus nephritis (regardless of activity) was associated with PD $(\mathrm{p}=0.00)$ and preeclampsia $(\mathrm{p}=0.03)$. Fortyfive women had a cesarean section and $37 \%$ of them were admitted with preterm labor. Median gestational age at birth was $37 \pm 5$ weeks and median birth weight was 2770 (710-4315) gr. There were two fetal deaths, two abortions and no maternal deaths.

Conclusion We present the first report of pregnancy outcomes of lupus patients in Uruguay. We highlight the low rate of complications, fetal and maternal death. It is possible that close, protocolized and multidisciplinary follow-up have a positive influence in these good results.

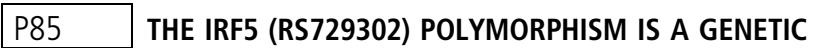 RISK FACTOR FOR SYSTEMIC LUPUS ERYTHEMATOSUS IN ALGERIAN PATIENTS}

${ }^{1}$ Ines Allam, ${ }^{2}$ Aldjia Lamri, ${ }^{2}$ Sihem Oulacrouz, ${ }^{2}$ Mohamed Saidani, ${ }^{1}$ Reda Djidjik. ${ }^{1}$ Dept. of Immunology, Beni Messous Hospital, Algiers; ${ }^{2}$ Dept. of nephrology, Beni Messous Hospital, Algiers, Algeria

\subsection{6/lupus-2020-eurolupus. 130}

Background Interferon regulatory factor 5 (IRF5) is a transcription factor regulating interferon secretion and was proved to be implicated in the pathogenesis of systemic lupus erythematosus (SLE) in several studies.

The purpose of this case-control study was to investigate whether IRF5 gene polymorphism is involved in the genetic predisposition to SLE in the Algerian population.

Methods IRF5 rs729302 (A/C) polymorphism was analyzed in 120 SLE patients and 98 age and sex matched controls by real time- polymerase chain reaction.

Results Significant association was observed for AA and AC genotypes of IRF5 between patients and healthy subjects $(60 \%$ vs $73 \% ; 40 \%$ vs $27 \%, \mathrm{p}=0.025$, respectively).
Patients with SLE had more frequent $\mathrm{C}$ allele compared to controls (20\% vs $13 \%, \mathrm{P}=0.041)$.

However, the allele and genotype frequencies did not show any difference in patients with nephritis in comparison to those without nephritis.

Conclusion The rs729302 C allele and AC genotype can be considered as risk factors for the development of SLE in Algerian patients.

\section{P86 THE NCF1-339 POLYMORPHISM IS ASSOCIATED WITH ALTERED FORMATION OF NEUTROPHIL EXTRACELLULAR TRAPS, HIGH SERUM INTERFERON ACTIVITY AND ANTIPHOSPHOLIPID SYNDROME IN SYSTEMIC LUPUS ERYTHEMATOSUS}

${ }^{1}$ Sabine Arve, ${ }^{1}$ Petrus Linge, ${ }^{2}$ Lina Olsson, ${ }^{3}$ Dag Leonard, ${ }^{4}$ Christopher Sjöwall, ${ }^{4}$ Martina Frodlund, ${ }^{5}$ Iva Gunnarsson, ${ }^{5}$ Elisabet Svenungsson, ${ }^{1}$ Helena Tydén, ${ }^{1}$ Andreas Jönsen, ${ }^{6}$ Robin Kahn, ${ }^{7}$ Åsa Johansson, ${ }^{3}$ Lars Rönnblom, ${ }^{2}$ Rikard Holmdahl, ${ }^{1}$ Anders Bengtsson. 'Dept. of Clinical Sciences, Rheumatology, Lund University, Lund; ${ }^{2}$ Dept. of Medical Inflammation Research, Section of Medical Biochemistry and Biophysics, Karolinska Institute, Stockholm; ${ }^{3}$ Dept. of Medical Sciences, Science for Life Laboratories, Rheumatology, Uppsala University, Uppsala; ${ }^{4}$ Dept. of Clinical and Experimental Medicine, Rheumatology, Linköping University, Linköping; ${ }^{5}$ Dept. of Medicine Solna, Rheumatology, Karolinska Institute and University Hospital, Stockholm; ${ }^{6}$ Dept. of Clinical Sciences, Pediatrics, Lund University, Lund; 'Dept. of Clinical Sciences, Hematology, Lund University, Lund, Sweden

\subsection{6/lupus-2020-eurolupus.131}

Background A single nucleotide polymorphism in NCF1 (NCF1-339, rs201802880), encoding NADPH oxidase complex 2 subunit $\mathrm{p} 47^{\text {phox }}$, reducing production of reactive oxygen species (ROS) is highly associated with development of systemic lupus erythematosus (SLE). However, the effect of NCF1-339 genotype on SLE regarding pathogenetic processes or comorbidities has not been investigated.

Methods NCF1-339 genotyped SLE subjects from four Swedish university hospitals were investigated regarding neutrophil ROS production $(n=31)$, neutrophil extracellular traps (NETs) $(\mathrm{n}=31)$, serum interferon $(\mathrm{n}=141)$, autoantibody profiles $(n=305)$ and clinical phenotypes $(n=1087)$.

Results Compared to SLE patients with normal-ROS NCF1339 genotypes, neutrophils from patients with low-ROS genotypes displayed impaired NET-formation and increased dependence on mitochondrial ROS for canonical NET-release. An increased frequency of low-ROS patients had high serum interferon activity. Patients with low-ROS genotypes had an increased frequency of positivity for antiphospholipid antibodies anti- $\beta 2$ glycoprotein I and anti-cardiolipin, related to the severe comorbidity antiphospholipid syndrome (APS). No other autoantibodies investigated were associated with NCF1339 genotype. Clinical characterization revealed a strong association between NCF1-339 low-ROS genotypes and secondary APS.

Conclusions NCF1-339 genotype affects neutrophil functions of ROS production, NET formation and dependence on mitochondrial ROS. SLE subjects with low-ROS NCF1-339 genotypes are associated with high serum interferon, presence of antiphospholipid antibodies and secondary APS.

Acknowledgements This study was primarily funded by FOREUM. 